\title{
The Prevention of Childhood Dental Caries in China
}

\author{
Xu Zheng ${ }^{1,2}$, Chun Yi Cao ${ }^{3}$, Sheng Ping Cao ${ }^{4}$, Qiuchi Ran ${ }^{1}$, Shi Wang Wu ${ }^{1}$, Zhu Ling Guo ${ }^{1,2, *}$ \\ ${ }^{1}$ School of Dentistry, Hainan Medical University, Haikou, PR China \\ ${ }^{2}$ Department of Dentistry, The First Affiliated Hospital of Hainan Medical University, Haikou, PR China \\ ${ }^{3}$ Hainan Eye Hospital and Key Laboratory of Ophthalmology, Zhongshan Ophthalmic Center, Sun Yat-sen University, Haikou, PR China \\ ${ }^{4}$ Institute of Clinical, Hainan Medical University, Haikou, PR China
}

Email address:

604569033@qq.com (Zhu Ling Guo)

${ }^{*}$ Corresponding author

\section{To cite this article:}

Xu Zheng, Chun Yi Cao, Sheng Ping Cao, Qiuchi Ran, Shi Wang Wu, Zhu Ling Guo. The Prevention of Childhood Dental Caries in China. American Journal of Pediatrics. Vol. 6, No. 3, 2020, pp. 228-233. doi: 10.11648/j.ajp.20200603.19

Received: May 16, 2020; Accepted: May 27, 2020; Published: June 4, 2020

\begin{abstract}
Children's oral health is the foundation of a healthy life. Dental caries is a common disease among children, which not only affects children's physical and mental health, but also has a negative impact on their families and the surrounding social environment. The world health organization (WHO) and the international dental union (FDI) have established a 2020 global goal for oral health. At present, the prevalence of dental caries among children in China is high, the awareness rate of oral health knowledge and behavioral development is low, the ability of oral health services at the grassroots level is weak, and the oral health work of children needs to be strengthened. This paper is a review paper and examines the basis of the disease and the recent studies carried out in various parts of The Peoples Republic of China, including the Hong Kong Special Administrative area. It recommended that oral hygiene be included in school curricula as part of children's general education, and called on Chinese teachers and parents to participate. At the same time, it is suggested that oral health care programmes be incorporated into general health education programmes and that children with dental caries be encouraged to take further active treatment in time.
\end{abstract}

Keywords: Dental Caries, Children, Dental Care, China

\section{Introduction}

Dental caries in children is a common and frequent oral disease because of the low degree of calcification of the milk teeth and the susceptibility to bacterial erosion. The etiology of childhood caries is a quadruple factor theory, which mainly includes bacteria, food, host and time. Although the primary cause of dental caries is due to cariogenic bacteria of which the primary causative ones are S. Mutans and Lactobacillus [1]. The basic point is that cariogenic foods, especially sucrose and refined carbohydrates are attached to the tooth surface. The acquired membrane formed by proteins from salvia is not only firmly attached to the tooth surface but also has sufficient time to form plaque. The deep layer produces acid which erodes, demineralizes, destroys the teeth, and then create cavities. Children's dental caries can affect their growth and development, cause psychological disorders, and oral and maxillofacial deformities and so on. The effects of dental caries in children are both widespread and far reaching and require further attention and the establishment of preventive dental programmes. This paper proposes that dental health education should be focused on the children's schools and special programmes should be set up which involve both the children and their parents and teachers. Methods involved should be both disclosing of individual children's teeth and intensive oral hygiene instruction.

\section{Childhood Oral Health Status}

The world health organization (WHO) and the international dental union (FDI) have established a global goal for oral health, including the rate of non-caries is less than $50 \%$ in children at the age of five to six [2]. However, according to national survey data, the prevalence rate of dental caries in Chinese 5-year-old children is as high as $71.9 \%$, while the relevant dental care index is no more than 3.6\% [3, 4]. As 
shown in Figure 1, from 2005 to 2015, Chinese primary teeth caries increase from $66.0 \%$ to $71.9 \%$, while permanent tooth caries increase from $28.9 \%$ to $38.5 \%$ [5]. Latest investigation further reveals that childhood dental caries increase from $70 \%$ to $75 \%$ between 5 -year-old and 6 -year-old children in China [6]. Although the prevalence of dental caries varies slightly in different regions and environments, the overall prevalence of dental caries is still high, even increasing. It is well known that the lack of awareness of oral hygiene in childhood and the lack of attention to dental cleanliness will increase the prevalence of dental caries and affect oral health in adulthood. Some studies have found that the oral health status of adolescents and elderly people in China is very poor, and compared with developed countries, the use level of oral health services in China is lower, which is closely related to the increasing prevalence of dental caries in children and periodontal diseases in adults [7, 8]. Adolescence is the transition and key stage from mixed dentition to permanent dentition, which will determine the basis of adult oral health [9]. Therefore, keeping good oral health behavior and paying attention to oral hygiene in childhood can effectively prevent the occurrence of dental caries and periodontal diseases, and provide certain guarantee for healthy oral state in adulthood.



Figure 1. The prevalence of primary and permanent dental caries in China from 2005 to 2015.

\section{Economic and Social Factors Affecting Dental Caries in Children}

Childhood caries begins early in life and progresses rapidly in those children who are at risk from the disease with consequences which can affect both the quality of life of the child and of the immediate family and may have wide ranging effects which extend both socially and economically beyond the family group [10]. Many studies have demonstrated that levels of education and the household income effect peoples' quality of life and social position and have therefore a direct effect on the level of dental caries found among the children in these groups [11, 12]. Because individuals have different levels of income and education, and their various oral conditions depend on dental floss, health care, toothbrushes, optional dental clinics, alternative treatments and preventive measures, and oral health knowledge. These differences directly lead to people with higher economic income and cultural level whose oral health behavior is significantly better than those with lower economic and cultural levels. So the educational level of fathers and mothers and their knowledge of oral health care have a significant impact on children's deciduous teeth [13].

For many young children, an emergency dental visit is their first dental visit and this may be either due to the discomfort or pain associated with dental caries. A significant proportion of the visits are due to early childhood caries, pathology associated with the oral soft tissues, problems with the eruption of adult teeth. Emergency dental care could be avoided by infant oral health and preventive dental programmes focused on parental education and early interventive dental treatment [14]. General lack of awareness of the importance of oral hygiene and regular visits to the dentist, causes dental caries still be the most epidemic disease in the twenty-first century. Early childhood caries can have a direct effect on the quality of life among the children and families involved. Damage caused by dental caries can affect the development of the dental system and cause acquired deformities. The pain of dental caries can further affect 
children's eating, due to they do not dare to chew with their sick teeth. Food without enough chewing will increase the burden of the stomach and cause stomach pain. Rough food cannot be digested completely in the stomach, which will affect the absorption of nutrients in the small intestine. Over time, it reduces the child's physical condition. If the dental caries is not treated in time, it will further develop inflammation of the pulp and cause unbearable pain. Long-term unilateral eating can affect the growth of the maxillofacial region and even a small deformity on one side of the face [15]. Therefore, the dental caries especially occurs in children should be treated in time.

China is a country in eastern Asia and is the most populous country in the world with a population of around 1.4 billion people. Since the introduction of economic reforms in 1978, China's economy has been one of the world's fastest-growing with annual growth rates consistently around $10 \%$, and has become the second-largest economy in the world now $[16,17]$ Due to the rapid development, many people have been uplifted out of poverty but many social issues still remain unsolved. One of these is malnutrition among rural children. The problem has diminished but still remains an important national issue [18]. And Many Chinese people are migrant workers and some travel large distances with their families to find work. Very few young children attend the dentist and there is a general lack of awareness about the need for regular dental care and the importance of oral hygiene.

In another aspect, Healthcare in China consists of both public and private medical institutions and insurance programs. About $95 \%$ of the population has at least basic health insurance coverage. Despite this, public health insurance generally only covers about half of medical costs, with the proportion lower for serious or chronic illnesses [19]. There are dental clinics in public hospitals and over the past few years private dental clinics have been established in many Chinese cities. Dental care is generally not covered by insurance so this means that children are attending the dentist only when they have been presenting problems as the costs of the treatment are paid directly by the children's parents. In some circumstances the parents will actively seek out the cheapest form of treatment and will not want to pay for preventive dental care. This can contribute to the prevalence of dental disease in children in some way.

\section{Dental Caries Among Chinese Children}

According to statistics, oral diseases have become a burden on global society and economy, especially in developing countries [20,21]. The incidence of dental caries is the second highest in the world, only followed by the upper respiratory tract infections [22]. Zhejiang is an eastern coastal province in China and is bordered by Jiangsu and Shanghai to the north, Anhui to the northwest, Jiangxi to the west and Fujian to the south. Data from Zhejiang province reveal that the average incidence of early childhood caries is $70.78 \%$ (Figure 2)
[23-25]. Wuhan is a city centrally placed in China and lying on the banks of the Yangtze River. Several studies have shown that the average prevalence of dental caries in primary dentition in children between 7 and 8 years old in Wuhan is $68.2 \%$ (Figure 2) [26, 27]. Investigations further indicates that the prevalence of dental caries could be reduced by the improvement of the oral insurance system, the enhancement of parents' awareness of children's dental care, and the timeliness of dental caries treatment.

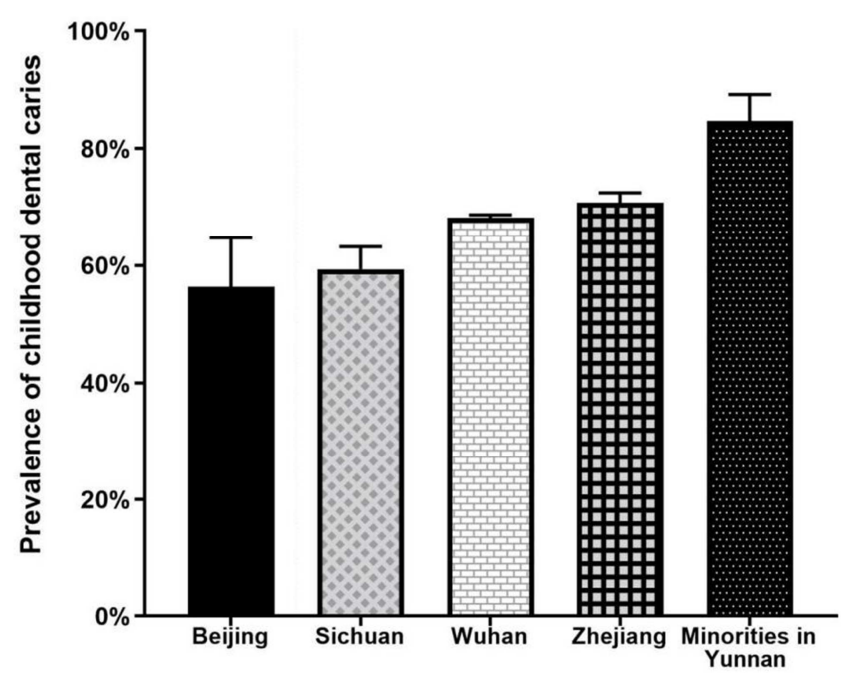

Figure 2. Prevalence of childhood dental caries in different regions of China.

Another study carried out in 2016 concentrated on the caries status of Lisu 400 preschool children in Yunnan Province, which is in south western China and leads on to the eastern end of the Himalayas [28]. The Lisu people are an ethnic minority group in China, and the average prevalence of dental caries among 5-year-old children was 80\% [29]. Similarly, as when compared to the other ethnic minorities of Yunnan with relatively poor medical resources, the rate of childhood dental caries in children is $85 \%$ in Bulang and $89 \%$ in Dai $[30,31]$. However, the prevalence of caries in the cities of Beijing and Sichuan in China is $56.33 \%$ and $59.36 \%$, respectively (Figure 2) [32-37]. Obviously, compared to the Chinese Han children (which is the largest ethnic Chinese group in China), the prevalence of caries among these ethnic groups was found to be higher. It is thought that the higher prevalence of caries in these areas may be due to limited medical resources [29, 30].

Hong Kong is a special administrative region of China. It had been a colony of the British Empire since 1842 and was returned to China in 1997. The Declaration between the British and Chinese guaranteed that Hong Kong would maintain its capitalist system and autonomous government for a period of 50 years after the transfer of sovereignty [38]. As shown in Figure 3, there was a high prevalence of dental caries among children in1968 and a significant improvement after fluoridation of the water supply. However, it wasn't long before the rising of dental caries rates found in pre-school children in Hong Kong [39, 40]. Thus the oral health in children in Hong Kong also needs significant improvement. 
The study puts forward the ideas that this should be in the form of education and combined with general health education [41]. With the higher incidence of dental caries among both in mainland and Hong Kong, further oral hygiene programmes should be encouraged throughout China and the prevention of dental caries in children should be based on prevention, starting from the cleaning aspect, and developing good dental care habits. It should be strengthened by the idea that the health education should be involved in "care for the teeth from a young age".

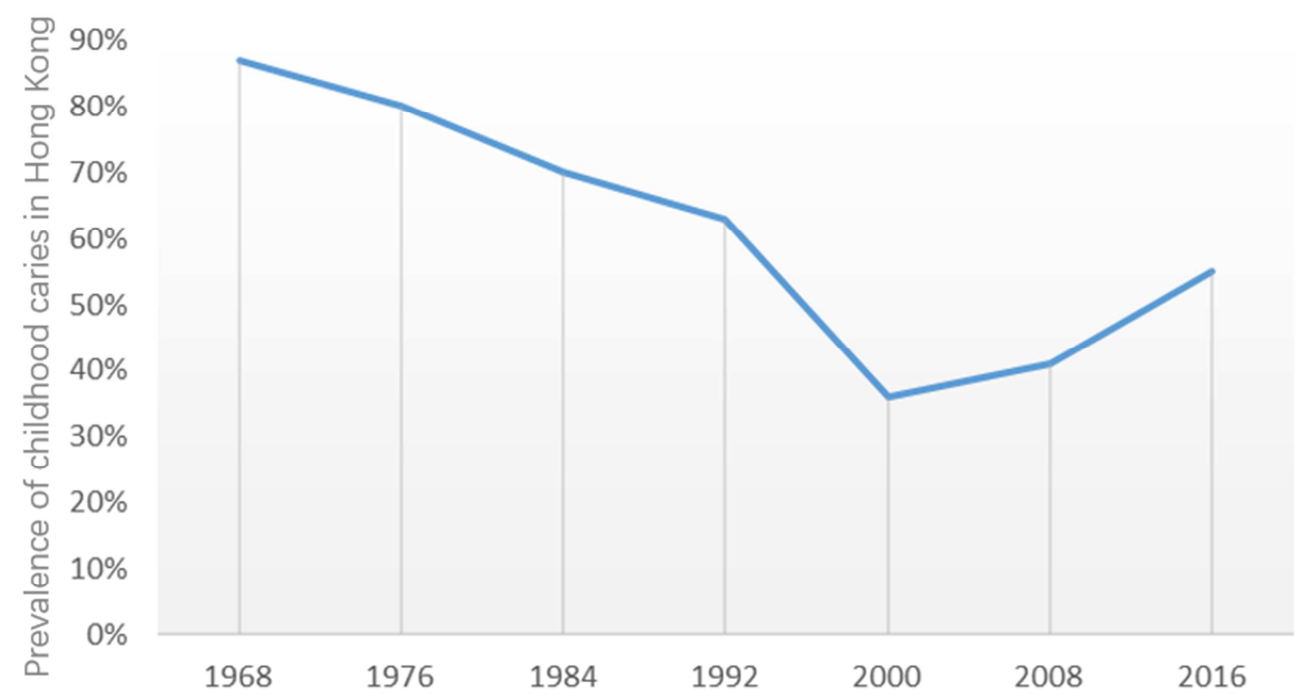

Figure 3. Early childhood caries in Hong Kong (1968-2016).

\section{Conclusion}

From the perspective of family, parents should strengthen the learning of oral hygiene knowledge and teach children by personal example as well as verbal instrution, so that it could cultivate a good family atmosphere taking oral hygiene seriously. In detail, parents should guide their children to develop proper oral hygiene habits, such as flossing correctly, brushing teeth correctly and visiting dentist regularly. Once caries or other dental diseases are found, parents should take their children to a regular hospital for treatment as soon as possible. From the point of society, oral hygiene programmes should be introduced into the school curriculum as a part of the children's general education, and the Chinese teachers should be involved in these programmes. In addition, the oral health care programmes can be incorporated into general health education programmes. Furthermore, some items such as oral health care and treatment of dental diseases can be considered to be covered by insurance in China, thus reducing the economic burden on people to maintain oral health.

\section{Acknowledgements}

This research was funded by Scientific Research Fund of Hainan Provincial Education Department (Hnky2020ZD-19), Young Talents' Science and Technology Innovation Project of Hainan Association for Science and Technology (QCXM201817), Medical Education Research Project of Medical Education Branch of Chinese Medical Association (2018B-N12003), Health Science Research Project of Hainan
Province (19A200062), Course Construction Project of Hainan Medical University (HYJW201834), Education Science Research Project of Hainan Medical University (HYZX201704), Marxism Theory Discipline Research Project of Hainan Medical University (QMYKYLX201807), Research Project of Hainan Provincial Humanities Medical Research Base (QRYZH201811 (YB)).

\section{References}

[1] Colak H, Dulgereill CT, Dalli M, et al. Early childhood caries update: A review of causes, diagnoses, and treatments [J]. Nat Sci Biol Med, 2013, 4: 29-38.

[2] Hobdell M, Petersen PE, Clarkson J, et al. Global goals for oral health 2020 [J]. Int Dent J, 2003, 53 (5): 285-288.

[3] Xiaoli Gao, Min Ding, Mengru Xu, et al. Utilization of dental services and associated factors among preschool children in China [J]. BMC Oral Health, 2020, 20: 9.

[4] Xiaonan Zhang, Sheng Yang, Zhaoying Liao, et al. Prevalence and care index of early childhood caries in mainland China: evidence from epidemiological surveys during 1987-2013 [J]. Sci Rep, 2016, 6: 18897.

[5] Wang Z, Rong W, Zhang Y, et al. Prevalence and contributing factors of dental caries of 6-year-old children in four regions of China [J]. Peer J, 2019.

[6] Chen KJ, Gao SS, Duangthip D, et al. Managing Early Childhood Caries for Young Children in China [J]. Healthcare, 2018, $6(1)$.

[7] Liu J, Zhang SS, Zheng SG, et al. Oral Health Status and Oral Health Care Model in China [J]. Chin J Dent Res, 2016, 19 (4): 207-215. 
[8] Cheng ML, Xu MR, Xie YY, et al. Utilisation of Oral Health Services and Economic Burden of Oral Diseases in China [J]. Chin J Dent Res, 2018, 21 (4): 275-284.

[9] Chen X, Ye W, Zhan JY, et al. Periodontal Status of Chinese Adolescents: Findings from the 4th National Oral Health Survey [J]. Chin J Dent Res, 2018, 21 (3): 195-203.

[10] Inglehart M R, Filstrup S L. Wander oral health and quality of life in children oral health-related quality of life [J]. Chicago: Quintessence Publishing Company, 2002, 5: 79-88.

[11] Locker D. Disparities in oral health- related quality of life in a population of Canadian children [J]. Community Dent Oral Epidemiol, 2007, 35 (3): 48-56.

[12] Jinghao $\mathrm{Hu}$, Wen Jiang, Xiaolong Lin, et al. Dental Caries Status and Caries Risk Factors in Students Ages 12-14 Years in Zhejiang, China [J]. Med Sci Monit, 2018, 24: 3670-3678.

[13] Philip W, Rick T, Deborah J, et al. Dental experiences and parenting practices of native American mothers and caretakers: What we can learn for the prevention of baby bottle tooth decay [J]. Dent Child, 1999, 66: 120-126.

[14] Andrea M de Silva, Shalika Hegde, Bridget Akudo Nwagbara, et al. Community-based population-level interventions for promoting child oral health [J]. Cochrane Database Syst Rev, 2016, 2016 (12).

[15] Livny A, Assali R, Sgan-Cohen H. Early childhood caries among a Bedouin community residing in the eastern outskirts of Jerusalem [J]. BMC Public Health, 2007, 7: 166-167.

[16] Sanmang Wu, Yalin Lei, Li Li. Evaluation of the Contributions of Four Components of Gross Domestic Product in Various Regions in China [J]. PLoS One, 2015, 10 (4): e0121594.

[17] Fu-Mei He, Tsangyao Chang, Zhen-Jiang Dou, et al. Non-linear Impact of China's Economic Growth on the Health of Residents-An Empirical Study Based on TVP-FAVAR Model [J]. Front Public Health, 2019, 7: 380.

[18] Park A, Wang S. China's Poverty Statistics [J]. China Economic Review, 2001, 12: 384-98.

[19] Yinzi Jin, Zhiyuan Hou, Donglan Zhang. Determinants of Health Insurance Coverage among People Aged 45 and over in China: Who Buys Public, Private and Multiple Insurance [J]. PLoS One, 2016, 11 (8): e0161774.

[20] Kailembo A, Preet R, Stewart Williams J, et al. Socioeconomic inequality in self-reported unmet need for oral health services in adults aged 50 years and over in China, Ghana, and India [J]. Int J Equity Health, 2018, 17 (1): 99.

[21] Petersen PE, Bourgeois D, Ogawa H, et al. The global burden of oral diseases and risks to oral health [J]. Bull World Health Organ, 2005, 83 (9): 661-669.

[22] Lu HX, Tao DY, Lo ECM, et al. The 4th National Oral Health Survey in the Mainland of China: Background and Methodology [J]. Chin J Dent Res, 2018, 21 (3): 161-165.

[23] Na Zhou, Haihua Zhu, Yadong Chen, et al. Dental caries and associated factors in 3 to 5 -year old children in Zhejiang province, China: an epidemiological study $[\mathrm{J}]$. BMC Oral Health, 2019, 19: 08-09.

[24] Xiping Wang, Zhiyuan Wei, Qiao Li, Liqin Mei. A longitudinal study of early childhood caries incidence in Wenzhou preschool children [J]. BMC Oral Health, 2017, 17: 105.
[25] Jiaqi Shao, mingyuan zou, rong fu, et al. Correlation between dental caries rate in preschool children and family background factors [J]. Electronic journal of general stomatology, 2018, 5 (09): 13-15 (In Chinese).

[26] Jin-Dong Wang, Xi Chen, Jo Frencken, et al. Dental caries and first permanent molar pit and fissure morphology in 7- to 8-year-old children in Wuhan, China [J]. Int J Oral Sci, 2012, 4 (3): $157-160$.

[27] Xuan Hu, Mingwen Fan, Jan Mulder, et al. Caries experience in the primary dentition and presence of plaque in 7-year-old Chinese children: A 4-year time-lag study [J]. J Int Soc Prev Community Dent, 2015, 5 (3): 205-210.

[28] Duangthip D, Gaoss Lo EC, Chu CH. Early childhood caries among 5 to 6 year old children in Southeast Asia [J]. Int Dent, 2017, 67: 98-106.

[29] Shinan Zhang, Yuexiao Li, Juan Liu, et al. Dental caries status of Lisu preschool children in Yunnan Province, China: a cross-sectional study [J]. BMC Oral Health, 2019, 19: 17.

[30] Zhang S, Liu J, Lo EC, ET AL. Dental caries status of Dai preschool children in Yunnan Province, China [J]. BMC Oral Health, 2013, 13: 67-68.

[31] Shinan Zhang, Juan Liu, Edward CM Lo, et al. Dental caries status of Bulang preschool children in Southwest China [J]. BMC Oral Health, 2014, 14: 16.

[32] Fan $\mathrm{C}$, Wang $\mathrm{W}, \mathrm{Xu} \mathrm{T}$, et al. Risk factors of early childhood caries among children in Beijing: a case-control study [J]. BMC Oral Health, 2016, 16: 98-99.

[33] Wei Yin, Ying-Ming Yang, Hong Chen, et al. Oral health status in Sichuan Province: findings from the oral health survey of Sichuan, 2015-2016 [J]. Int J Oral Sci, 2017, 9 (1): 10-15.

[34] LI Ke-zeng, LI Xue, HU De-yu, et al. Prevalence of deciduous tooth caries in 780 childr en aged 5 years [J]. West China journal of stomatology, 2008 (01): 70-72 (In Chinese with English abstract).

[35] Mengru Xu, Chao Yuan, Xiangyu Sun, et al. Oral health service utilization patterns among preschool children in Beijing, China [J]. BMC Oral Health, 2018, 18: 31.

[36] Can Can Fan, Wen Hui Wang, Tao Xu, et al. Risk factors of early childhood caries (ECC) among children in Beijing - a prospective cohort study [J]. BMC Oral Health, 2019, 19: 34.

[37] WANG Zhuo, DENG Ying, LIU Shi Wei, et al. Prevalence and Years of Life Lost due to Disability from Dental Caries among Children and Adolescents in Western China, 1990-2015 [J]. Biomedical and Environmental Sciences, 2017, 30 (10): 701-707.

[38] Summers T. The Hong Kong special administrative regionat 20; evaluating the sino-British joint declaration [J]. Asian Education and Development Studies, 2017, 7: 89-101.

[39] Wang Wc, Chen Jh, Chen PH, et al. Significant caries and the interactive effects of maternal-related oral hygiene factors in urban preschool children [J]. Public Health Dent, 2017, 77: 188-196.

[40] Duangthip D, Chen KJ, Gao SS, et al. Early childhood caries among 3- to 5-year-old children in Hong Kong [J]. Int Dent J, 2019, 69 (3): 230-236. 
[41] Chen K J, Gao S S, Duangthip D, et al.. Early childhood caries and oral health care of Hong Kong preschool children [J]. Clinical, Cosmetic and Investigative Dentistry, 2019, 11: 27-35. 